\title{
EXTENDED X-RAY ABSORPTION FINE STRUCTURE IN CATALYSIS
}

\author{
A.J. RENOUPREZ \\ Institut de Recherches sur la Catalyse \\ 2, av. Albert Einstein, 69626 Villeurbanne cedex, France
}

\begin{abstract}
Applications of extended X-ray absorption fine structure and X-ray edge structure to catalysis are described. Special attention is paid to the study of supported mono- and bimetallic catalysts. The method used to collect in situ data, under reaction conditions are also considered.
\end{abstract}

PACS numbers: 61.10.Lx, 82.65.Jv

\section{Introduction}

During the last ten years extended X-ray absorption fine structure (EXAFS) and related X-ray absorption methods have proved to be powerful tools to characterize catalytic materials. Two reasons can be invoked. First, it is simultaneously a spectroscopic and a structural probe. Compared to diffraction, it has the unique advantage that one can know around which atom the local order is determined. Second, it has a high sensitivity, largely ascribable to the considerable photon flux of the modern synchrotrons.

Among the properties of EXAFS which makes it useful for the study of catalysts are:

- long-range order is not required and most of the catalytic systems do not contain well-ordered phases,

- the local atomic arrangement can be determined around each type of atom separately by tuning the photon energy,

- the structural information is obtained by a simple analysis of the data with an accuracy comparable to that obtained by diffraction on single crystals,

- the data collection is so fast that kinetic measurements are now currently performed,

- in situ measurements under reaction conditions can be performed. 


\section{Theory of EXAFS and data analysis}

\subsection{Theory}

The fundamental discovery of EXAFS was actually performed in 1971 by Stern and Sayers [1] who showed that the oscillations of the X-ray absorption coefficient located at an energy higher than the absorption edge of a given element are related to the short-range order around this element.

They derived a formula which expresses that the Fourier transform of the EXAFS signal is a radial distribution function which peaks at distances corresponding to the successive coordination shells of atoms.

When X-ray photons have enough energy to free a bound electron in the atom, an absorption edge is observed: $K$ - or $L$-edges for $1 s$ or $2 p$ electrons. The kinetic energy of the ejected photoelectron is the difference between the photon energy and the binding energy of electron.

For photon energies 15-20 eV above the edge, only the final state of the photoelectron is perturbed by the surroundings. This final state is modified by a single scattering from each surrounding atom and is the superposition of outgoing and scattered waves. This phenomenon is represented in Fig. 1.

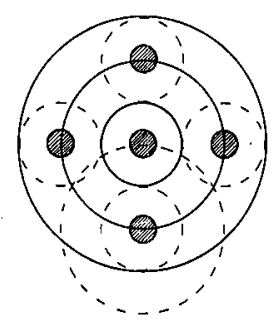

Fig. 1. Schematic representation of the interference between outgoing wave (solid line) and backscattered wave (dashed line).

The outgoing wave has an amplitude proportional to $\exp (\mathrm{i} k r) / r$, where $k=2 \pi / \lambda$ and $\lambda$ is the wavelength. The amplitude of the backscattered wave, characteristic of the backscattering atom, reads at $r_{i}$, position of this atom,

$$
A_{i}(2 k) \frac{\exp \left(\mathrm{i} k r_{i}\right)}{r_{i}} \frac{\exp \left[\mathrm{i} k\left(r-r_{i}\right)\right]}{r-r_{i}} \text {. }
$$

At the origin $(r=0)$ the wave has travelled the distance $2 r_{i}$ and its amplitude is proportional to

$$
A(2 k) \frac{\exp \left(\mathrm{i} 2 k r_{i}\right)}{r_{i}^{2}}
$$

Actually, the electrons do not travel in a constant potential and both central and scattering atoms add an additional phase shift to the wave and the amplitude becomes

$$
A_{i}(2 k) \operatorname{expi}\left[2 k r_{i}+\delta(k)\right]
$$


The interference between backscattered and outgoing waves modifies the absorption coefficient $\chi_{i}(k)$ :

$$
\chi_{i}(k)=\frac{K A_{i}(2 k)}{r_{i}^{2}} \sin \left[2 k r_{i}+\delta_{i}(k)\right]
$$

the real part of $(3)$.

Two other factors modify the absorption coefficient. First, the photoelectron has a finite lifetime because of scattering from surrounding atoms. It is taken into account by a mean free path term $\exp \left(-2 r_{i} / L\right)$, where $L$ is a mean free path of the photoelectron, in general a function of $k$. Also in a coordination shell, all the atoms will not be at the same distance from the central atom, because of thermal vibrations and static disorder. If this disorder has a Gaussian distribution, it will add a factor $\exp \left(-2 k^{2} \sigma_{i}^{2}\right)$, where $\sigma_{i}$ is the relative mean square deviation from the distance $r_{i}$. The final EXAFS formula becomes, when extended to all the atoms of the various shells,

$$
\chi(k)=\sum_{i} \frac{N_{i}}{k R_{i}^{2}} A_{i}(k) \exp \left(-2 R_{i} / L\right) \exp \left(-2 \sigma^{2} k^{2}\right) \sin \left[2 k R_{i}+\delta_{i}(k)\right] .
$$

A Fourier transform of $\chi(k)$ will thus lead to the number of atoms $N_{i}$ in the shell located at $R_{i}$ from the central atom. The nature of these atoms can be identified because $A(k)$ is characteristic of a given backscattering element.

\subsection{Data analysis}

The experimental absorption coefficient $\mu(E)$ has to be normalized first to the atomic absorption $\mu_{0}(E)$ to obtain $\chi(E)$ :

$$
\chi(E)=\frac{\mu(E)-\mu_{0}(E)}{\mu_{0}(E)} .
$$

This subtraction is performed by approximating first the experimental data by a simple polynomial followed by digital high-pass filtering. The resulting curve is then subtracted from the experimental one. The successive steps of the data reduction are shown in Fig. 2.

The second step consists in converting $\chi(E)$ to $\chi(k)$ with

$$
k=\left[\frac{2 m}{k^{2}}\left(E-E_{0}\right)\right]^{1 / 2},
$$

where $E_{0}$ is the energy of the edge. Note that the choice for $E_{0}$ will have a marked influence on $R_{i}$, the radius of a shell.

The phase shifts contained in expression (5) were computed for a given pair of atoms by McKale [2] together with the backscattering amplitude of all the elements. One can also make use of experimental parameters measured on a compound of known structure.

The fit of expression (5) to the experimental data should thus lead to $N$ and $R$ for the various shells; however, $N$ and $\sigma$ (the Debye-Waller term) are not independent variables. Since the thermal part of $\sigma$ decreases at low temperature, 

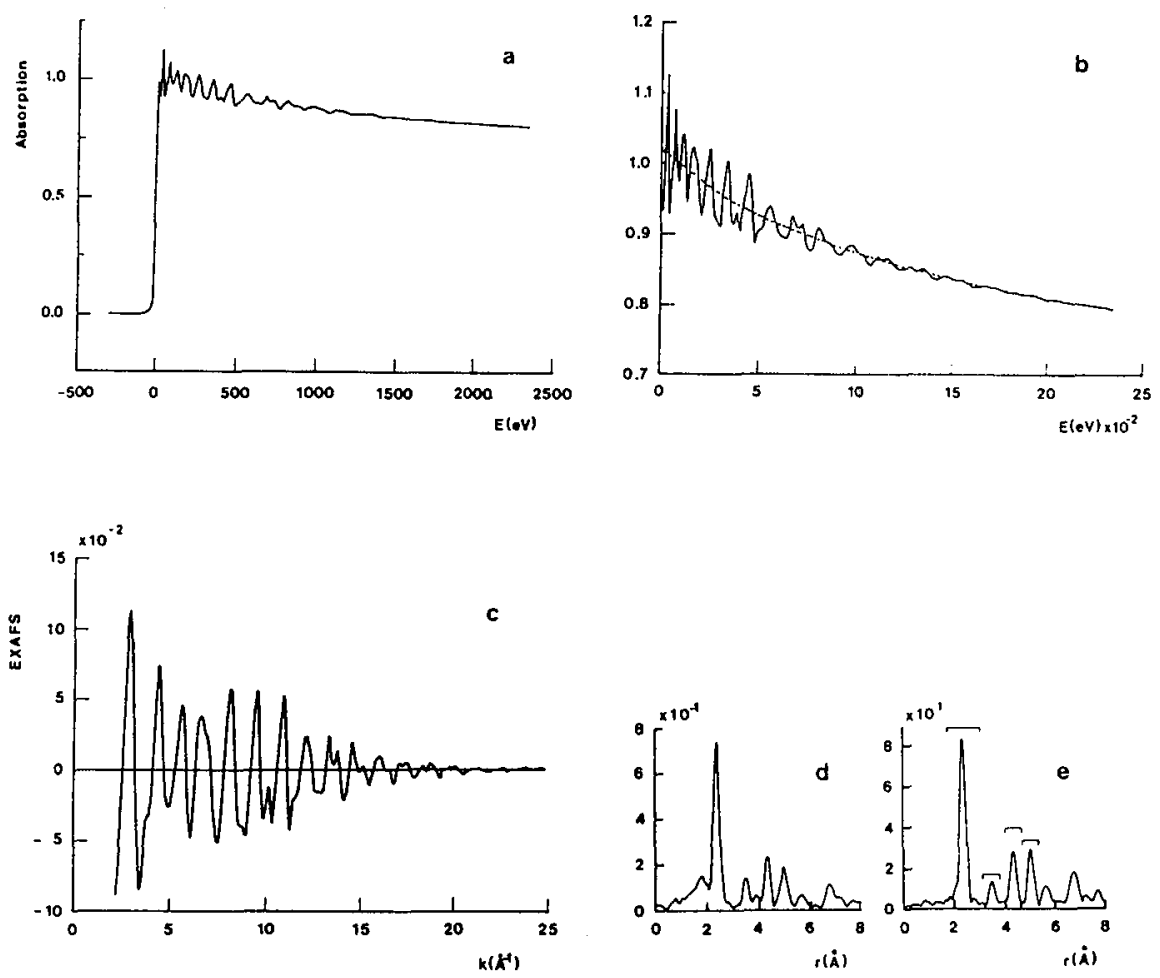

Fig. 2. Illustration of the various steps of the data treatment. (a) Raw adsorption spectrum of a $\mathrm{Rh}$ foil at the $\mathrm{Rh} K$-edge $(23.320 \mathrm{eV})$. (b) The same data as in (a) on an extended scale, above the edge. The background used to extract the EXAFS is the dashed line. (c) The normalized EXAFS after background subtraction. (d) and (e) $k^{1}$ and $k^{3}$ weighted Fourier transforms of (c). Four successive shells are visible in (e).

a more accurate determination of $N$ would be obtained by low-temperature experiments.

Usually one isolates first each shell on the Fourier transform of the EXAFS signal and adjusts it to a model. If the statistics is of the order of $10^{-4}$ and if the data extends over a large $k$ range $\left(0-18 \AA^{-1}\right)$, the accuracy of $R$ is $\pm 0.02 \AA$ and $15 \%$ of $N$.

An excellent review on the various problems of data analysis is presented in Ref. [4].

\section{Experimental}

\subsection{Synchrotron radiation}

At the synchrotron facility, the photons are produced by high-energy electrons or positrons $(500 \mathrm{MeV}$ to $6 \mathrm{GeV})$ travelling in a storage ring. 
The broad wavelength distribution has a maximum of intensity close to a critical value

$$
c=5.59 \frac{R_{\mathrm{m}}}{E^{3}}
$$

where $R_{\mathrm{m}}$ is the radius of curvature of the bending magnet and $E$ - the energy of the electrons in $\mathrm{GeV}$. The total scattered power $P_{\mathrm{t}}$ is

$$
P_{\mathrm{t}}=88.5 \frac{E^{4} I}{R} \text {. }
$$

The interesting features of the synchrotron radiation are:

- a flux 5-6 orders of magnitude larger than with X-ray tubes, typically $10^{14}$ photons $\cdot \mathrm{s}^{-1} \cdot \mathrm{mrad}^{-2}$ for $0.1 \%$ band width,

- a large range of energies: $2-50 \mathrm{keV}$ at the European Facility (ESRF),

- a high brilliance resulting from the small source size $(0.1-0.2 \mathrm{~mm})$ and low vertical divergence of the beam ( $0.1 \mathrm{mrad})$.

This last point, together with the width of the rocking curve of the monochromator, determines the energy resolution.

\subsection{The instrument}

The EXAFS instrument is composed of three main parts:

1. The X-ray optics.

2. The detection system.

3. The sample environment.

The X-ray optics is composed of a mirror which delivers a parallel beam and removes the high-energy photons when necessary. The mirror is followed by a two crystal monochromator which is rotated to scan the energies. Silicon crystals with (111), (200) or (311) orientations are generally used; they have an energy resolution of $2 \times 10^{-4}$ to $10^{-5}$.

The detection system is composed of a first counter which measures $I_{0}$, the photon flux before the sample, and a second detector measuring $I$, the intensity behind it. The absorption coefficient is

$$
\mu(E)=\log \frac{I_{0}}{I} .
$$

$I$ and $I_{0}$ are usually measured by ion chambers, a type of counter limited at low flux by its noise and at high flux by charge accumulation effects resulting in a loss of linearity.

As a function of the available flux, one has to vary the nature of the gas in the counters and its pressure to optimize the efficiency.

Teo [3] showed that the signal to noise ratio $(\mathrm{S} / \mathrm{N})$ is given for thick samples by the expression

$$
\mathrm{S} / \mathrm{N}=0.557 \frac{\mu_{\mathrm{A}}}{\mu_{\mathrm{T}}}\left(\frac{\Delta \mu_{\mathrm{A}}}{\mu_{\mathrm{A}}} I_{0}^{1 / 2}\right)
$$


where $\mu_{\mathrm{T}}=\mu_{\mathrm{A}}+\mu_{\mathrm{B}}, \mu_{\mathrm{A}}$ is the absorption coefficient of the studied element, $\mu_{\mathrm{B}}$ - of the matrix, $\Delta \mu_{\mathrm{A}} / \mu_{\mathrm{A}}$ is the EXAFS signal. The optimum sample thickness, $X$, occurs at $\mu_{\mathrm{T}} X=2.56$. For thin samples

$$
\mathrm{S} / \mathrm{N}=\mathrm{e}^{-\mu_{\mathrm{B}} X_{\mathrm{B}} / 2} \mu_{\mathrm{A}}\left(\frac{\Delta \mu_{\mathrm{A}}}{\mu_{\mathrm{A}}} I_{0}^{1 / 2}\right)
$$

It can easily be seen that absorption measurements can be difficult if $\mu_{\mathrm{B}} X_{\mathrm{B}}$ is large, i.e. if for example the catalyst is supported on a highly absorbing material. This is the case if the active element has a low $Z$ number and thus an edge located at low energy. It will be for example quite impossible to study by transmission diluted samples composed of $\mathrm{Fe}$ or $\mathrm{Cr}$ supported on $\mathrm{ZrO}_{2}$. On the contrary, it will be easy with $\mathrm{Pd} / \mathrm{SiO}_{2}$, since the $\mathrm{Pd} K$-edge is located at $24 \mathrm{keV}$, an energy where $\mu_{\mathrm{B}}$ for $\mathrm{SiO}_{2}$ is low.

Typically, with a photon flux of $10^{12}$ photons $/ \mathrm{s}, \Delta \mu_{\mathrm{A}} / \mu_{\mathrm{A}} \sim 10^{-2}, \mu_{\mathrm{A}} / \mu_{\mathrm{T}}=$ $10^{-3}$, the signal to noise ratio will be of the order of $10^{2}$ in $1 \mathrm{~s}$. Thus, the time of experiment for a $1 \mathrm{keV}$ scan and $2 \mathrm{eV}$ step is of the order of $10 \mathrm{~min}$.

Many effects worsen the results and, first, the nonlinearity and saturation of the ion chambers. For this reason photodiodes are now preferred; they have a low dark current and remain linear for photon flux larger by two orders of magnitude than the gas detectors.

For the study of diluted elements a good alternative to transmission detection is fluorescence. Indeed, the excited absorber atom with a core hole, relaxes by undergoing a radiative transition. The fluorescence yield near the edge is proportional to the absorption coefficient. This fluorescence yield varies with $Z$, for example from 0.35 for $\mathrm{Fe}$ to 0.96 for $\mathrm{Pt}$.

For example, an EXAFS experiment at the $\mathrm{Fe} K$-edge located at $7.112 \mathrm{keV}$ is performed by collecting the photons corresponding to $K_{\alpha_{1}}, K_{\alpha_{2}}$, and $K_{\beta}$ emissions located at $6.403,6.39$ and $7.057 \mathrm{keV}$. To improve the $\mathrm{S} / \mathrm{N}$ in fluorescence, one has to remove the elastic photons. A good solution is to use a filter composed of the $Z-1$ element. In the above example, the elastic photons have an energy comprised, during the scan, between 7.1 and $8 \mathrm{keV}$ and a Mn filter with a $K$-edge located at $6.54 \mathrm{keV}$ will be used. Scintillation detectors equipped with plastic scintillators can collect up to $3 \times 10^{6}$ photons/s. One also uses solid state detectors which have a good energy resolution ( $200 \mathrm{eV}$ at $10 \mathrm{keV}$ ) but are limited in counting rate to $10^{4} \mathrm{imp}$./s. The multicell Ge detector manufactured by Camberra is composed of 13 individual diodes. It has an energy resolution of $160 \mathrm{eV}$ at $6 \mathrm{keV}$ and a counting rate close to $10^{5} \mathrm{imp} . / \mathrm{s}$.

Less expensive solutions will certainly appear soon. They will be composed of multistrip Si or Ge detectors with 100 to 200 strips with fully integrated electronics.

\subsection{Sample environment}

Catalytic systems are generally composed of finely divided metals or sulphides which can undergo transformations when exposed to atmosphere. Moreover, it is highly desirable to investigate structure modifications, poisoning etc. under catalytic conditions. For these reasons, many authors have designed high-temperature cells [4] where the catalysts can be heated up to $900 \mathrm{~K}$ under gas 
flow and cooled down to $100 \mathrm{~K}$ to minimize the Debye-Waller factor. Some of them can work both in transmission and fluorescence mode.

In a recent achievement [8], such a cell was coupled with a chromatographic detection to study under pressure $(10 \mathrm{bar})$ the cocking of a catalyst during a reforming reaction.

\section{EXAFS on metallic catalysts}

\subsection{Monometallic catalysts}

A large number of studies have been performed on metallic supported catalysts during the last ten years; this interest is due to the importance of the oil reforming catalytic process. EXAFS was able to elucidate the various steps of the catalysts preparation and of their deactivation under catalytic conditions.

A good example is the study of the preparation of $\mathrm{Pd} /$ alumina catalysts known to be selective in the hydrogenation of dienes to alcenes.

Lesage [5] showed that when $\alpha$-alumina is impregnated with $\left(\mathrm{C}_{5} \mathrm{H}_{7} \mathrm{O}_{2}\right)_{2} \mathrm{Pd}$, the $\mathrm{Pd}$ atom is surrounded by 4 oxygen atoms located at $1.96 \AA$. After drying at $100^{\circ} \mathrm{C}$, one finds 5 oxygen atoms at $1.98 \AA$; this corresponds to the loss of one ACAC molecule and to the coordination of the $\mathrm{Pd}-\mathrm{C}_{5} \mathrm{H}_{7} \mathrm{O}_{2}$ residue to 3 oxygen atoms of the alumina. Calcination up to $700^{\circ} \mathrm{C}$ progressively leads to the formation of oxide and the observation of Pd-Pd pairs in the second coordination shell.

A large number of studies were also devoted to the study of reforming catalysts composed of $\mathrm{Pt} / \gamma$-alumina [6, 7]. Special attention was paid to the influence of chlorine on the formation of the catalysts.

In the study by Guyot-Sionnest [8], a non-chlorinated and a chlorinated compound $(1 \% \mathrm{Pt}, 2 \% \mathrm{Cl})$ were compared. It was shown that after the calcination treatment in air at $500^{\circ} \mathrm{C}$ for non-chlorinated samples, the first coordination sphere is that of $\beta-\mathrm{PtO}_{2}$ with 6 oxygen atoms surrounding platinum. But in the second and third shells composed of $\mathrm{Pt}-\mathrm{Pt}$ pairs at 3.2 and $3.5 \AA$, the coordination numbers are $1 / 2$ those of $\mathrm{PtO}_{2}$. This indicates that the oxide particles have a very small size. When $\mathrm{Cl}$ is present in the sample, $\mathrm{Cl}$ atoms are observed in the first shell and the numbers of $\mathrm{Pt}$ atoms in the second and third shell are still reduced.

During catalyst reduction in flowing $\mathrm{H}_{2}$, the number of Pt-O begins to decrease at $150^{\circ} \mathrm{C}$ to reach a stable value of 0.3 at $460^{\circ} \mathrm{C}$. Simultaneously, Pt-Pt first neighbours appear above $200^{\circ} \mathrm{C}$ to reach 5.4 for $\mathrm{Pt} / \mathrm{Al}_{2} \mathrm{O}_{3}$ and 4.1 for $\mathrm{Pt} / \mathrm{Al}_{2} \mathrm{O}_{3}-\mathrm{Cl}$ at $460^{\circ} \mathrm{C}$.

Such low coordination numbers of 4 to 5.5 compared to 12 in bulk materials mean that the particles are composed of 10-15 atoms. The comparison of the Fourier transform (FT) of $\mathrm{PtO}_{2}$ and of the catalysts is shown in Fig. 3.

An important point should be outlined: in this type of study, precise values for the backscattering amplitude and phase shift parameters are needed. For this, model compounds with known crystallographic parameters are studied by EXAFS. For the $\mathrm{Pt}-\mathrm{O}$ pair $\beta-\mathrm{PtO}_{2}$ was used, for the $\mathrm{Pt}-\mathrm{Cl}, \mathrm{H}_{2} \mathrm{PtCl}_{6}$ and a $\mathrm{Pt}$ foil for the Pt-Pt pair. 

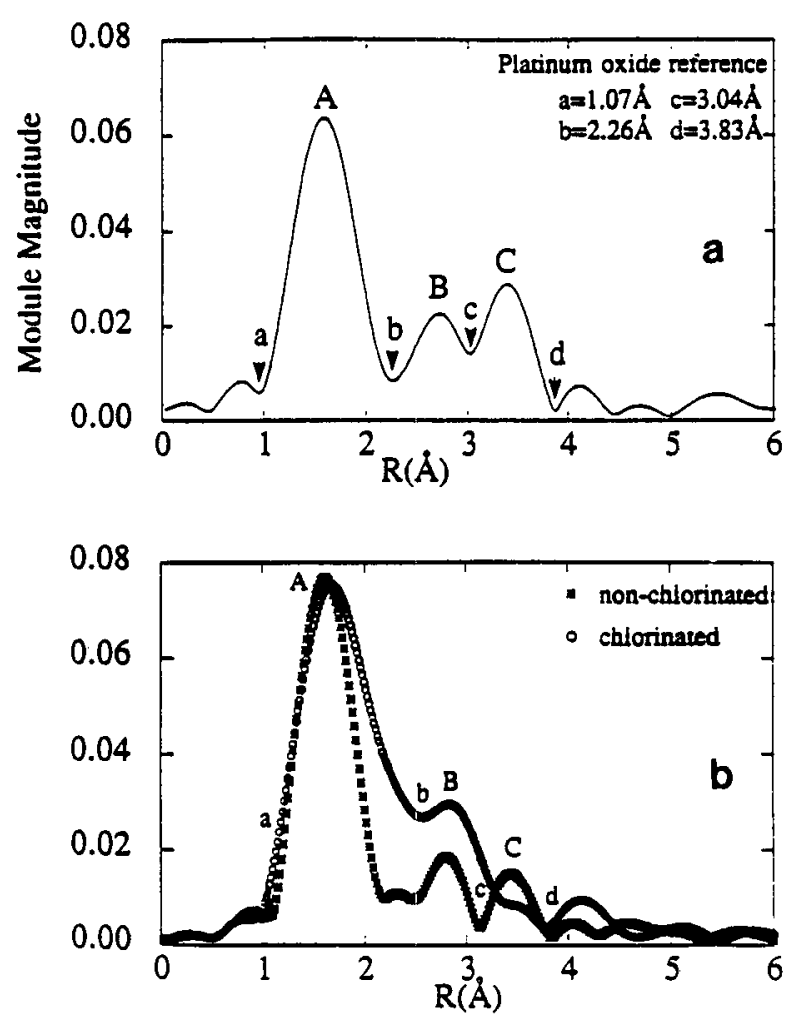

Fig. 3. FT of $\mathrm{PtO}_{2}$ reference compound (a) and two $\mathrm{Pt} / \mathrm{Al}_{2} \mathrm{O}_{3}, 1$ wt\% chlorinated and non-chlorinated (b) (from Ref. [8]).

Actually, these in situ experiments performed at high temperature suffer from a limited $k$ range, typically $10 \AA^{-1}$; the consequence is that the accuracy of $R$ is certainly not larger than $0.05 \AA$.

A catalytic conversion of $N$-heptane was also performed on these catalysts in an EXAFS cell. During the various reactions occurring at $350^{\circ} \mathrm{C}$, the formation of $\mathrm{Pt}-\mathrm{C}$ bonds are observed. After the reaction, $\mathrm{Pt}$ is surrounded by $1.4 \mathrm{C}$ atoms at $1.96 \AA$ and $5 \mathrm{Pt}$ atoms at $2.67 \AA$. Flowing hydrogen at $450^{\circ} \mathrm{C}$ for $3 \mathrm{~h}$ removes a large fraction of the carbon ( $0.4 \mathrm{Pt}-\mathrm{C}$ only). The successive transformations of the catalyst are illustrated in Fig. 4.

Another example of a complete structure determination of a metallic supported catalyst was performed for Pd/Y-zeolite [6]. These $10 \AA$ particles encaged in the large cavity of the zeolite were studied at $100 \mathrm{~K}$. Because of the low temperature, the data extend up to $18 \AA^{-1}$ and the first three layers could be analysed (Figs. 5 and 6, Table I). It was concluded that the particles are composed of $45 \pm 5$ atoms arranged in truncated octahedron with fcc symmetry. 

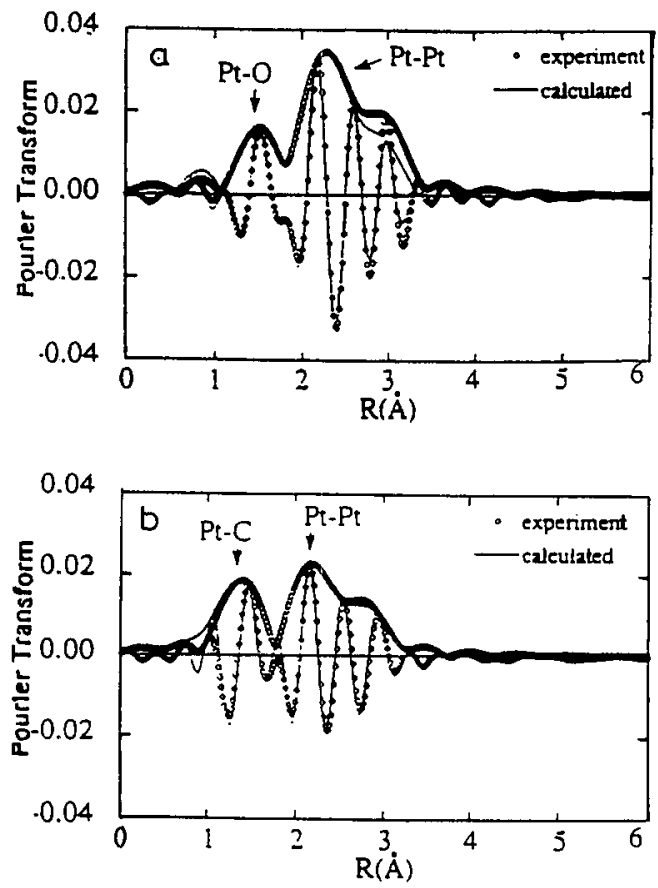

Fig. 4. $\mathrm{Pt} / \mathrm{Al}_{2} \mathrm{O}_{3}$ during the reduction treatment (a) and after cataly tic conversion (b) of $N$-heptane at $460^{\circ} \mathrm{C}$.

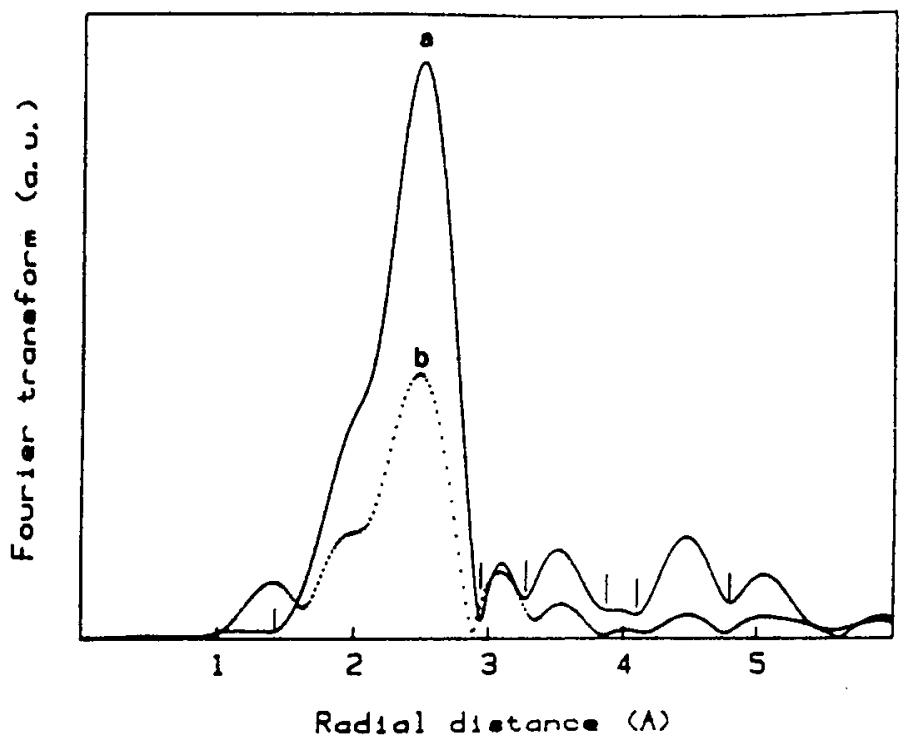

Fig. 5. Fourier transform of a Pd foil (a) and $2 \% \mathrm{Pd}$ in Y zeolite (b). Note the reduction of amplitude of the peak on the FT because of the small particle size (from Ref. (6). 


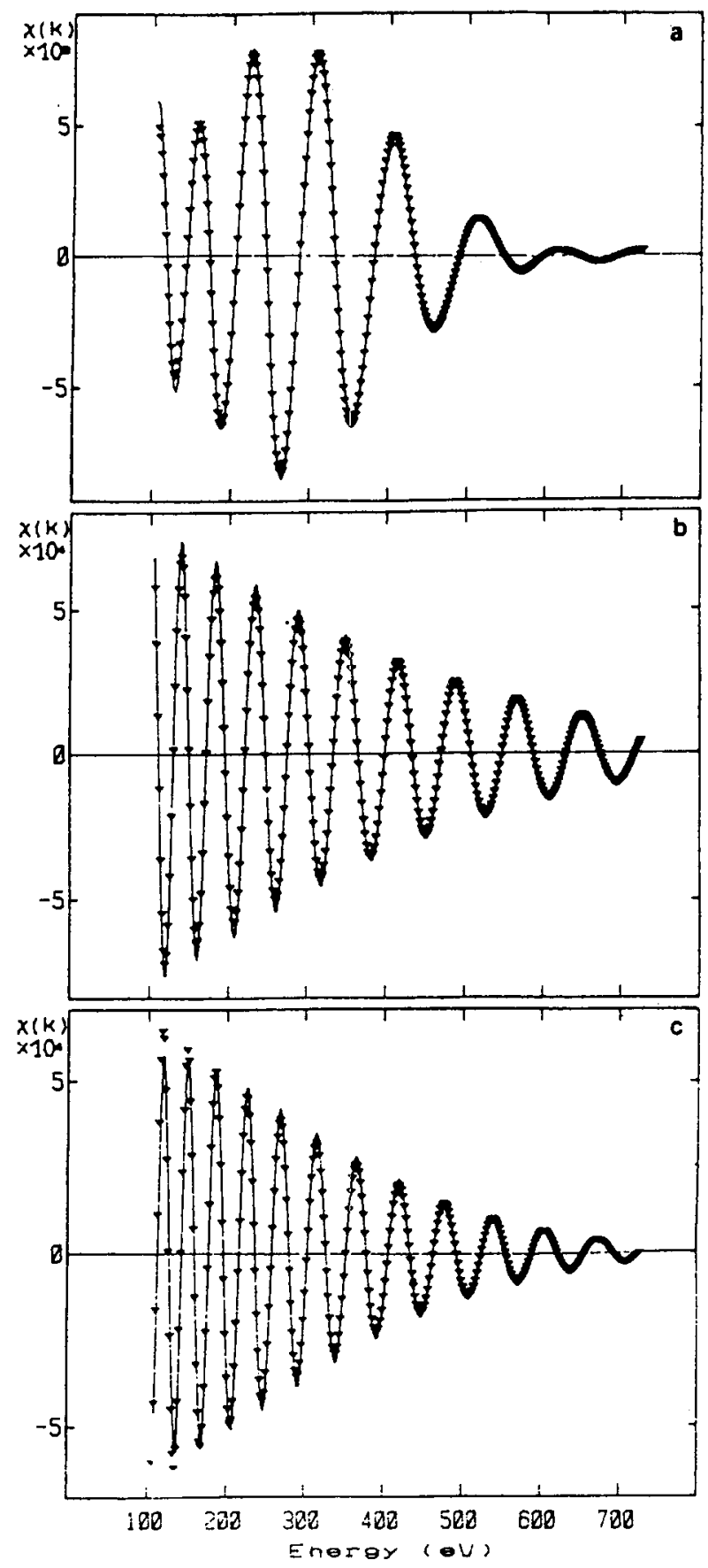

Fig. 6. Fit of the first 3 peaks of the FT of Fig. $5 \mathrm{~b}$ with models. The best fit corresponds to a $4 \mathrm{O}$ atoms truncated octahedron. 
TABLE I

$\mathrm{Pd} / \mathrm{Y}$-zeolite. Comparison between the coordination numbers of the first three layers with those of models.

\begin{tabular}{l|c|c|c|c|c|c|c}
\hline \hline & $\begin{array}{c}N \\
\text { atoms }\end{array}$ & $\begin{array}{c}R_{1} \\
{[\AA]}\end{array}$ & $N_{1}$ & $R_{2}$ & $N_{2}$ & $R_{3}$ & $N_{3}$ \\
{$[\AA]$} & & {$[\AA]$} & \\
\hline Pd foil & - & 2.75 & 12 & 3.89 & 6 & 4.76 & 24 \\
Pd/zeolite & - & 2.73 & 7.5 & 3.92 & 2.5 & 4.79 & 9.2 \\
Cubooctahedron & 54 & - & 7.85 & - & 3.27 & - & 9.6 \\
Octahedron & 44 & - & 7.63 & - & 2.6 & - & 8.7 \\
Truncated octahedron & 38 & - & 7.57 & - & 2.7 & - & 8.8
\end{tabular}

\subsection{Bimetallic catalysts}

Modern reforming catalysts are now composed of platinum in association with other elements such as Re, Sn, Ge, Ir, etc. The characterization of small bimetallic particles requires a probe with an atomic resolution. One needs to answer the following questions:

- Are the two metals fully reduced?

- Is a true alloy formed by these two metals?

- Is there any surface segregation?

Obviously, the complete answer necessitates experiments at both edges. The analysis of the EXAFS data is more complex than in the previous case because, generally, model compounds containing both elements are not available.

In the well-known case of $\mathrm{Ru}-\mathrm{Cu} / \mathrm{SiO}_{2}$ studied by Simfeld [9] (Fig. 7) the phase shifts $\delta_{\mathrm{Cu}-\mathrm{Ru}}$ and $\delta_{\mathrm{Ru}-\mathrm{Cu}}$ are obtained by measuring, first, Ru and Cu edges of $\mathrm{RuO}_{2}$ and $\mathrm{CuO}$ and then,

$$
\delta_{\mathrm{Ru}-\mathrm{Cu}}=\delta_{\mathrm{RuO}}+\delta_{\mathrm{Cu}-\mathrm{Cu}}-\delta_{\mathrm{CuO}}
$$

and

$$
\delta_{\mathrm{Cu}-\mathrm{Ru}}=\delta_{\mathrm{RuO}}+\delta_{\mathrm{Ru}-\mathrm{Ru}}-\delta_{\mathrm{CuO}} .
$$

It was found that the coordination numbers in the first shell are, respectively, 9 and 11 for $\mathrm{Cu}$ and $\mathrm{Ru}$. This indicates that $\mathrm{Cu}$ with lower coordination number migrates at the surface of the particle.

Ilowever, the chemical analysis corresponds to a $1: 1$ ratio of $\mathrm{Cu}$ to $\mathrm{Ru}$ but one finds $92 \%$ of $\mathrm{Ru}$ atoms around $\mathrm{Ru}$ and $50 \% \mathrm{Cu}$ atoms around $\mathrm{Cu}$ with distances $\mathrm{Ru}-\mathrm{Ru}=2.65 \AA$ on $\mathrm{Cu}-\mathrm{Cu}=2.58 \AA$. This confirms that $\mathrm{Ru}$ atoms are in the core of the particle with mainly Ru atoms around them and that $\mathrm{Cu}$ has a mixed neighbourhood with $\mathrm{Ru}$ atoms probably lying in the second layer below the surface.

These observations are in line with oxygen chemisorption measurements which show that only $\mathrm{Cu}$ is oxidized and that the $\mathrm{CuO}$ layer protects the underlying $\mathrm{Ru}$ atoms. 

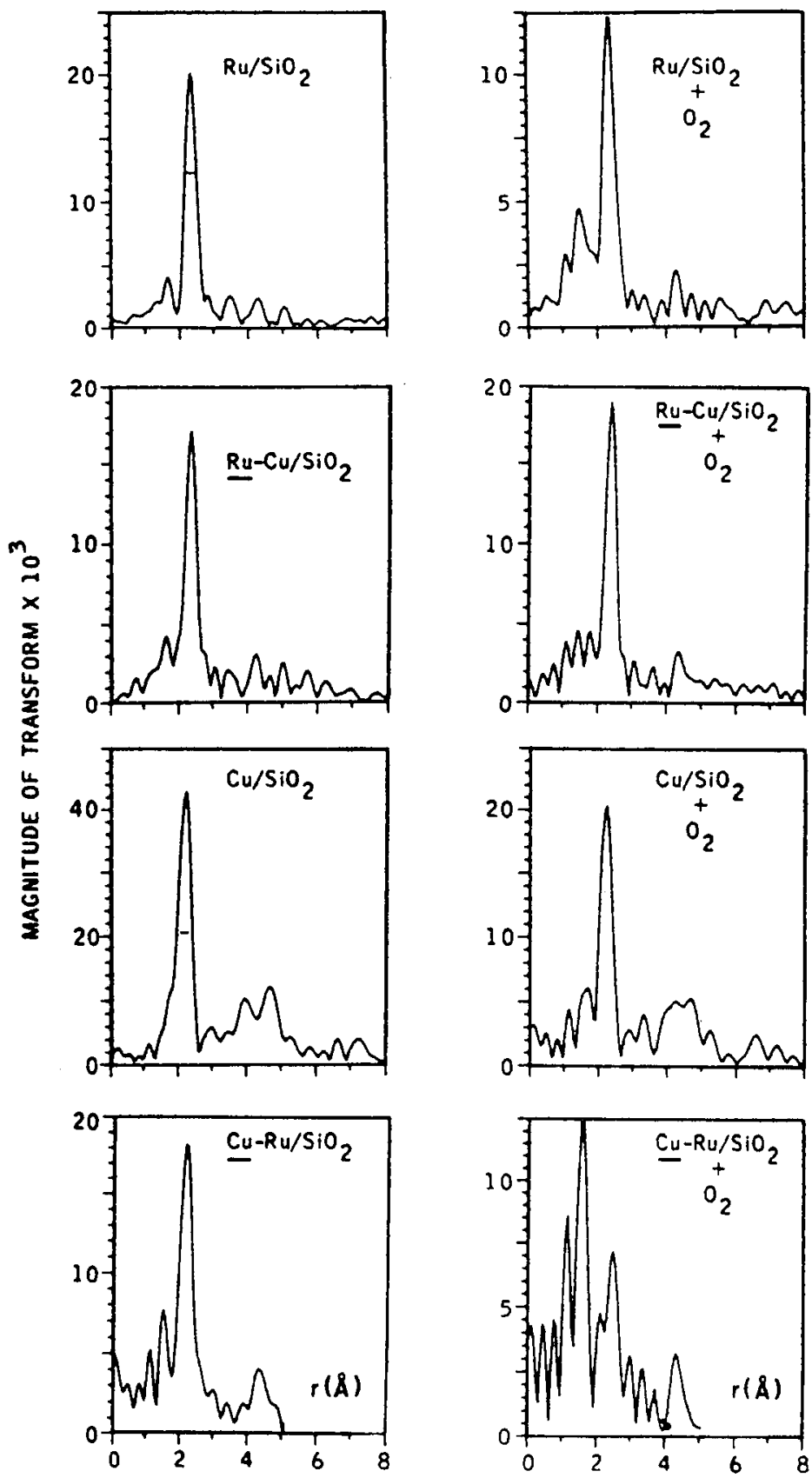

Fig. 7. FT of $\mathrm{Ru}, \mathrm{Cu}$ and $\mathrm{Ru}-\mathrm{Cu} / \mathrm{SiO}_{2}$ catalysts before (left) and after (right) exposure to oxygen. The reduction of amplitude of the peak of the FT at the $\mathrm{Cu} K$-edge and not at the $\mathrm{Ru}$ edge upon $\mathrm{O}_{2}$ adsorption, demonstrates that $\mathrm{Cu}$ is at the surface of the particle (from Ref. [9]). 
White lines observed at adsorption edges are attributed to transition from $2 p$ to $n d$ states. For elements with uncompleted bands, the photoelectron is ejected to empty levels close to the Fermi level, resulting in an increase in the adsorption coefficient, a few $\mathrm{eV}$ above the edge. This was used by many authors [10] to probe the chemical state of this element. Upon exposure to oxygen, $\mathrm{Pt}, \mathrm{Ir}, \mathrm{Rh}$ exhibit a strong increase in the $L_{\mathrm{III}}$ line compared to the $L_{\mathrm{II}}$ which can be related to creation of vacancies.

This type of probe can also be used to investigate more subtle electronic structure modifications which occur in bimetallic systems. A good example is the study of $\mathrm{Pt} L_{\mathrm{III}}$-edge of $\mathrm{Pt}-\mathrm{Fe}$ bimetallic catalysts performed by Moraweck et al. [11].

It was shown that upon increasing the $\mathrm{Fe}$ concentration the $5 d$ holes in $\mathrm{Pt}$ bands are progressively filled by iron electrons. This can be related to modifications of the catalytic properties.

\section{Hydrodesulphurization catalysts}

The study of hydrodesulphurization catalysts used in the treatment of crude oils constitutes the best example of the success of X-ray adsorption in the structure determination of complex systems $[12,13]$. They are constituted of molybdenum oxides supported on alumina associated with a promoter composed of cobalt oxides.

In the oxidized state, the $\mathrm{Co}-\mathrm{Mo} / \mathrm{Al}_{2} \mathrm{O}_{3}$ catalyst contains cobalt atoms surrounded by 4 oxygen atoms at $1.99 \AA$ like in $\mathrm{CoAl}_{2} \mathrm{O}_{4}$ and Mo is surrounded by 6 oxygen atoms at $1.81 \AA$.

Upon sulphurization of the catalyst, Co is now surrounded by $4 \mathrm{O}$ at $2.03 \AA$ and $6 \mathrm{~S}$ at $2.33 \AA$. The Co-O distance is too short compared to that found in $\mathrm{CoO}(2.14 \AA)$ and too large for a Co in tetrahedral sites as in $\mathrm{CoAl}_{2} \mathrm{O}_{4}$ (Table II). Actually, this can be interpreted by the presence of two types of cobalt species; the first one has a coordination of 6 as in $\mathrm{CoS}$, the second one with $4 \mathrm{O}$ atoms would anchor the particle to $\mathrm{Al}_{2} \mathrm{O}_{3}$.

TABLE II

Environment of Co and Mo in model compounds and in $\mathrm{Co}-\mathrm{Mo} / \mathrm{Al}_{2} \mathrm{O}_{3}$.

\begin{tabular}{c|c|c|c|c}
\hline \hline \multirow{2}{*}{ Sample } & \multicolumn{2}{|c|}{ Co edge } & \multicolumn{2}{c}{ Mo edge } \\
\cline { 2 - 5 } & $N$ & $R[\AA]$ & $N$ & $R[\AA]$ \\
\hline $\mathrm{CoMoO}_{4}$ & $6 \mathrm{O}$ & 2.09 & $6 \mathrm{O}$ & 1.98 \\
$\mathrm{MoS}_{2}$ & - & - & $6 \mathrm{~S}$ & 2.41 \\
$\mathrm{Co}-\mathrm{Mo}_{\mathrm{Al}_{2} \mathrm{O}_{3}}$ & $4 \mathrm{O}$ & 1.99 & $6 \mathrm{~S}$ & 2.29 \\
$+\mathrm{S}$ & $6 \mathrm{~S}$ & 2.29 & - & -
\end{tabular}

The investigations at the Mo edge showed that Mo is surrounded by $6 \mathrm{~S}$ at $2.37 \AA$ as in $\mathrm{MoS}_{2}$ but in the second sphere only 4 Mo atoms at $3.25 \AA$ are found 
instead of 6 in $\mathrm{MoS}_{2}$. This is the indication of the presence of small (10 $\AA$ ) $\mathrm{MoS}_{2}$ clusters.

The promoting effect of Co in these catalysts can be attributed to an electron transfer from Co-S bonding orbitals to antibonding Mo-S. An increase in electron density on Mo-S would enhance the activation of $\mathrm{H}_{2}$.

\section{Conclusions}

$\mathrm{X}$-ray absorption is now one of the most powerful analytical tool to study the structure of catalysts. Moreover, the last versions of quick EXAFS or dispersive EXAFS are able to follow the kinetics of structural modifications occurring during catalytic reactions. Its ability to probe the various elements constituting the catalyst is also a precious help to optimize its preparation.

\section{References}

[1] D.E. Sayers, E.A. Stern, F.W. Lytle, Phys. Rev. Lett. 27, 1204 (1971).

[2] A.G. McKale, B.W. Veal, A.P. Paulikas, S.K. Chan, G.S. Knapp, J. Am. Chem. Soc. 110, 3763 (1988).

[3] B.K. Teo, in: EXAFS Spectroscopy, Eds. B.K. Teo, D.C. Joy, Plenum Press, New York 1979, p. 13.

[4] X-Ray Adsorption, Chemical Analysis, Vol. 92, Eds. D.C. Koningsberger, R. Prins, Wiley, New York 1988.

[5] E. Lesage-Rosenberg, Ph.D. thesis, Technip, 1984.

[6] B. Moraweck, A. Renouprez, Surf. Sci. 106, 35 (1981).

[7] B. Moraweck, G. Clugnet, A. Renouprez, J. Chim. Phys. 83, 265 (1986).

[8] N. Guyot-Sionnest, Ph.D. thesis, LURE, Orsay 1991.

[9] T.H. Sinfeld, G.H. Via, R.B. Greegor, J. Chem. Phys. 75, 5527 (1981).

[10] F.W. Lytle, J. Catal. 43, 376 (1976).

[11] B. Moraweck, P. Bondot, D. Goupil, P. Fouilloux, A. Renouprez, J. Phys. (France) 8, 39, 263 (1986).

[12] G. Sankar, S. Vasudevan, C.N.R. Rao, J. Phys. Chem. 91, 2011 (1987).

[13] B.S. Clausen, H. Topsoe, R. Candia, T. Villadsen, B. Lengeler, T. Alsnielsen, T. Christensen, J. Phys. Chem. 85, 3868 (1981). 